\section{P-017 DELAYED CEREBRAL ISCHEMIA SECONDARY TO ARTERIOVENOUS MALFORMATION RUPTURE}

K Amuluru, F Al-Mufti, C Prestigiacomo, C Gandhi. Neurological Surgery and Neuroscience, Rutgers University - New Jersey Medical School, Newark, NJ

\subsection{6/neurintsurg-2016-012589.59}

Objective Vasospasm and resultant clinical deterioration caused by delayed cerebral ischemia (DCI) are well known sources of morbidity after aneurysmal subarachnoid hemorrhage. Cerebral arteriovenous malformation (AVM) rupture is a relatively common cause of spontaneous parenchymal hemorrhage, as well as subarachnoid and intraventricular hemorrhage. However, ensuing vasospasm associated with AVM rupture is rare, with only 20 cases documented in the literature. We report a series of patients with ruptured AVMs in order to evaluate the prevalence of vasospasm and resultant DCI.

Methods We retrospectively reviewed our own series of 854 patients presenting to our institution with an ICD-9 code for intracranial hemorrhage between September 2005 and May 2014. Thirty-six of these patients were found to have acute intracranial hemorrhage secondary to a ruptured cerebral AVM. Diagnostic cerebral angiograms were reviewed by two blinded neurointerventionalists. Electronic medical records, laboratory data, all related noninvasive imaging and angiographic data of the patients were reviewed.

Results The mean age of the patients was 43 years; there was a 1.25:1 male to female predominance. Twenty-six patients had an intraparenchymal hemorrhagic component (72\%), while 10 patients $(28 \%)$ had pure extra-axial hemorrhage. Fifteen patients had subarachnoid component (42\%) and 24 patients had intraventricular component (67\%). Three patients $(8.3 \%)$ had pure intraventricular hemorrhage. All patients had AVMs diagnosed on CT angiography or digital subtraction angiography as part of their workup for the intracranial hemorrhage. All patients had digital subtraction angiography within 7 days of hemorrhage. Twenty-seven patients had supratentorial AVM nidus. Five patients demonstrated angiographic vasospasm (13.9\%), 4 of whom went on to develop DCI (11.1\%). The mean time from ictus to demonstration of vasospasm was 6.6 days. All of the 5 patients who developed vasospasm had intraventricular hemorrhage. Of the 3 patients with pure intraventricular hemorrhage, all developed DCI.

Conclusions Although typically associated with aneurysmal subarachnoid hemorrhage, angiographic vasospasm after AVM rupture is possible. AVM rupture classically produces parenchymal hemorrhage, but may also present with subarachnoid and/or intraventricular hemorrhage, both of which are associated with vasospasm and DCI. In cases with AVM rupture causing extra-axial hemorrhage, arterial vasospasm may be a rare, however under-reported, consequence. Specifically, in cases with pure intraventricular hemorrhage, arterial vasospasm does remain a possibility and may be a factor in patients who develop DCI.

Disclosures K. Amuluru: None. F. Al-Mufti: None. C. Prestigiacomo: None. C. Gandhi: None.

\section{P-018 PARENT ARTERY CURVATURE AND ANGLE OF INCIDENCE OF BLOOD FLOW AFFECT FLOW DIVERSION EFFECT OF PIPELINE}

${ }^{1} \mathrm{~S}$ Tateshima, ${ }^{2} \mathrm{R}$ Sagas, ${ }^{2} \mathrm{C}$ Kularni, ${ }^{2} \mathrm{D}$ Duong, ${ }^{2} \mathrm{~S}$ Obi. ${ }^{1}$ Interventional Neuroradiology, Ronald Reagan UCLA Medical Center, Los Angeles, CA; ${ }^{2}$ Keio University Department of Mechanical Engineering, Yokohama, Japan

\subsection{6/neurintsurg-2016-012589.60}

Introduction Pipeline flow diversion stent is often placed in a tortuous anatomy. Nevertheless, effect of parent artery curvature on the flow diversion effect from Pipeline stent is poorly understood. The flow diversion effect could be enhanced or diminished depending on the curvature of Pipeline or the angle at which blood flow strikes the Pipeline (angle of incidence). These variations may explain why not all aneurysms are obliterated after Pipeline treatment despite its high efficacy. We evaluated the flow diversion effect of Pipeline in various parent artery curvatures and angle of incidence in invitro models.

Methods Four acrylic prototypes of curved tube with a side branch were constructed with circular cross section with the inner diameter of $3.8 \mathrm{~mm}$. The variation among the prototypes was made in terms of the radius of curvature $\mathrm{R}$ $(\mathrm{R}=25 \mathrm{~mm}$ and $50 \mathrm{~mm}$ ), and the relative angle $\theta$ of the side branch $(\theta=60,90$, and 120). The angle $\theta$ of the side branch determines the angle of incidence. The identical Pipeline of diameter $4.75 \mathrm{~mm}$ were placed to each prototype and the pressure loss $(\mathrm{K})$ occurring in the flow through the Pipeline mesh was measured for the Reynolds numbers ranging from 100 to 800 . Careful error analysis on the measured data
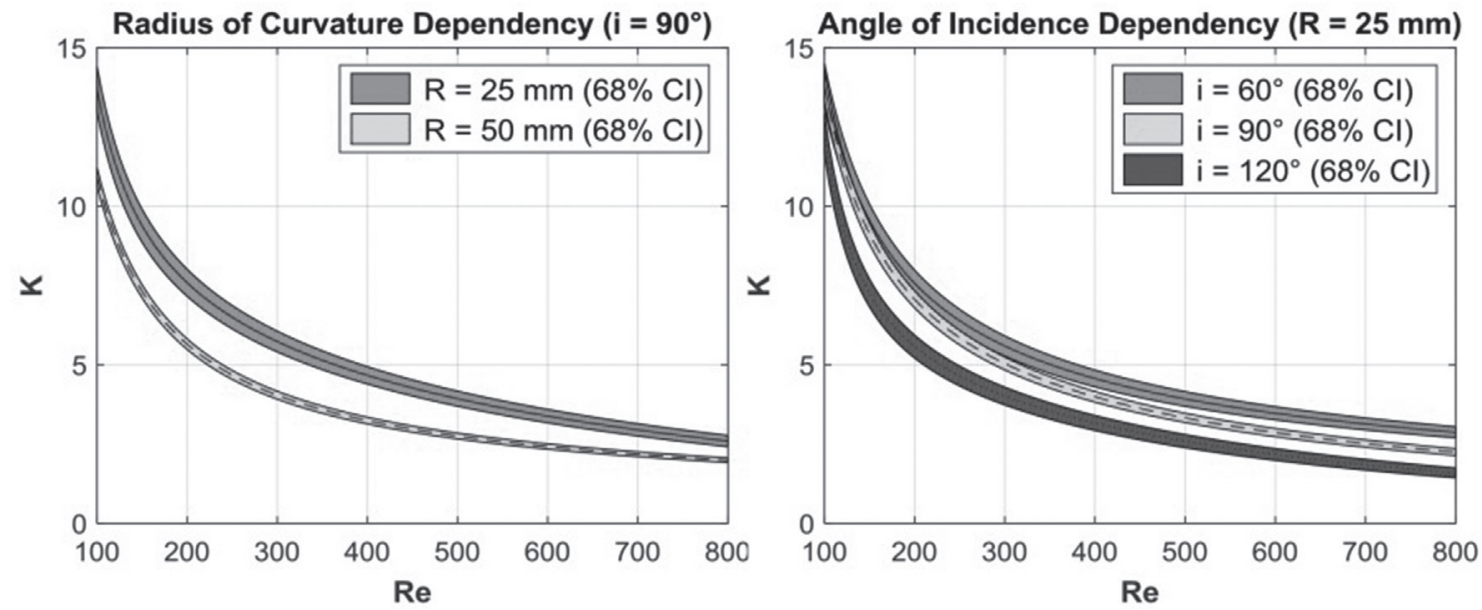

Abstract P-018 Figure 1 